\title{
BMJ Open Assessing relative COVID-19 mortality during the second wave: a prospective Swiss population-based study
}

\author{
Sandra Siegfried (D) , ${ }^{1}$ Matthias Bopp, ${ }^{1}$ Huldrych Günthard, ${ }^{2,3}$ Olivia Keiser, ${ }^{4}$ \\ Caroline E Weibull, ${ }^{5}$ Michael Crowther, ${ }^{6}$ Torsten Hothorn (i) ${ }^{1}$
}

To cite: Siegfried S, Bopp M, Günthard $\mathrm{H}$, et al. Assessing relative COVID-19 mortality during the second wave: a prospective Swiss populationbased study. BMJ Open 2021;11:e051164. doi:10.1136/ bmjopen-2021-051164

- Prepublication history and additional supplemental material for this paper are available online. To view these files, please visit the journal online (http://dx.doi.org/10.1136/ bmjopen-2021-051164).

Received 11 March 2021 Accepted 16 September 2021

\section{Check for updates}

(C) Author(s) (or their employer(s)) 2021. Re-use permitted under CC BY-NC. No commercial re-use. See rights and permissions. Published by BMJ.

${ }^{1}$ Institut für Epidemiologie, Biostatistik und Prävention, Universität Zürich, Zürich,

Switzerland

${ }^{2}$ Institut für Medizinische Virologie, Universität Zürich, Zürich, Switzerland

${ }^{3}$ Klinik für Infektionskrankheiten und Spitalhygiene,

Universitätsspital Zürich, Zürich, Switzerland

${ }^{4}$ Institut de santé globale, Université de Genève, Geneva, Switzerland

${ }^{5}$ Department of Medicine Solna, Karolinska Institutet, Stockholm, Sweden

${ }^{6}$ Department of Medical Epidemiology and Biostatistics, Karolinska Institutet, Stockholm, Sweden

Correspondence to Professor Torsten Hothorn; Torsten.Hothorn@uzh.ch

\section{ABSTRACT}

Objective During the first COVID-19 wave in Switzerland, relative mortality was at least eight times higher compared with the uninfected general population. We aimed to assess sex-specific and age-specific relative mortality associated with a SARS-CoV-2 diagnosis during the second wave.

Design Prospective population-based study.

Setting Individuals testing positive for SARS-CoV-2 after the start of the second wave on 1 October 2020 were followed up until death or administrative censoring on 31 December 2020.

Participants 5179740 inhabitants of Switzerland in fall 2018 aged 35-95 years (without COVID-19) and 257288 persons tested positive for SARS-CoV-2 by PCR or antigen testing during the second wave.

Primary and secondary outcome measures The planned outcome measure was time to death from any cause, measured from the date of a SARS-CoV-2 diagnosis or 1 October in the general population. Information on confirmed SARS-CoV-2 diagnoses and deaths was matched by calendar time with the all-cause mortality of the general Swiss population of 2018. Proportional hazards models were used to estimate sex-specific and agespecific mortality rates and probabilities of death within 60 days.

Results The risk of death for individuals tested positive for SARS-CoV-2 in the second wave in Switzerland increased at least sixfold compared with the general population. HRs, reflecting the risk attributable to a SARSCoV-2 infection, were higher for men $(1.40,95 \% \mathrm{Cl} 1.29$ to 1.52) and increased for each additional year of age (1.01, $95 \% \mathrm{Cl} 1.01$ to 1.02 ). COVID-19 mortality was reduced by at least 20\% compared with the first wave in spring 2020 . Conclusion General mortality patterns, increased for men and older persons, were similar in spring and in fall. Absolute and relative COVID-19 mortality was smaller in fall.

Trial registration The protocol for this study was registered on 3 December 2020 at https://osf.io/gbd6r.

\section{INTRODUCTION}

An overwhelming body of research has been published since the start of the SARS-CoV-2 pandemic in early 2020. Many reports provide assessments of the burden of COVID-19 from multiple perspectives. The frequency of fatal

\section{Strengths and limitations of this study}

- Relative sex-specific and age-specific COVID-19 mortality rates for the second wave of fall 2020 comparing a cohort of COVID-19 patients with the general population are provided.

- A direct comparison with the relative mortality reported for the first wave in spring 2020 is possible, without confounding by differences in reporting systems, public health interventions or demographics.

- The present prospectively planned study replicates the main findings reported in a retrospective analysis of Swiss surveillance data from the first wave.

- The study excludes patients younger than 35 years and older than 95 years, as well as patients tested posthumously, and information on relevant comorbidities or sociodemographic information is not available at the population level.

- Fatal outcomes were studied exclusively, no assessment of the burden of COVID-19 in terms of hospitalisations or long COVID was possible.

outcomes is most commonly measured by case fatality rates, ${ }^{1-3}$ infection fatality rates ${ }^{45}$ or excess number of deaths. ${ }^{67}$ Early estimates have been challenged and updated ${ }^{8-11}$ during and after the first wave in spring 2020. Case and infection fatality rates have been criticised because interpretation depends on public health measures taken, testing and reporting systems, assessment of cause of death and underlying demographic characteristics, most importantly age distributions. Thus, fatality rates are hard to compare between different populations and in the same population over time ${ }^{12-15}$ and adjustment for the age composition and sex composition of the population is crucial. ${ }^{16-19}$

Longitudinal assessments of the burden of disease became more important during the onset of second waves in summer and fall 2020. Comparisons of case fatality rates in the first and second wave have been published, ${ }^{20}{ }^{21}$ indicating lower fatality rates 
during the second wave, but contrary results have also been reported. ${ }^{22}$

Excess mortality allows longitudinal patterns of COVID-19 mortality to be compared with the general population mortality without confounding by demographic characteristics and public health interventions. ${ }^{23}$ Analysis of the all-cause mortality does not require the differentiation between deaths caused by COVID-19 or other reasons and might thus allow for a more comprehensive picture of the burden of disease. ${ }^{24-26}$

An additional measure to assess the burden of disease is the relative mortality, which is defined as the ratio in absolute probabilities of death within a given time frame, across two different populations. The sex-specific and age-specific relative COVID-19 mortality was reported for the first wave of spring 2020 in Switzerland, ${ }^{27}$ where mortality was at least eight times higher compared with the uninfected population. Sex and age had little impact on this relative risk.

In this study, we present sex-specific and age-specific relative COVID-19 mortality during the second wave in fall 2020 in Switzerland and based on these results compare relative COVID-19 mortality between the first and the second wave. In contrast to the first wave in Switzerland, which was characterised by strict public health interventions and moderate number of deaths, ${ }^{28}$ the second wave caused local exceedances of hospital capacity and more than five times the number of COVID-19-related deaths, following late implementation of moderate public health interventions.

\section{METHODS}

\section{Study design}

This population-based study was planned prospectively (see study protocol) and includes information of two cohorts: individuals tested positive for SARS-CoV-2 in the second wave in Switzerland (Swiss Fall 2020 COVID-19 cohort) and the general Swiss population of fall 2018 (Swiss Fall 2018 cohort).

\section{Data sources}

The population-based data for Switzerland from 2014 to 2019 were obtained from the Swiss Federal Statistical Office, including daily death records and age-specific numbers of male and female inhabitants on 31 December of each year.

Records of individuals tested positive for SARS-CoV-2 from 1 October 2020 to 31 December 2020, including dates of tests and occurred deaths, were provided by the Swiss Federal Office for Public Health (FOPH). In Switzerland, SARS-CoV-2 tests were performed either by PCR and, since 2 November 2020, additionally with rapid antigen tests of lower and upper respiratory tract samples. ${ }^{29}$ Test results were reported to the FOPH. Persons with positive SARS-CoV-2 tests were followed up. The testing recommendations in Switzerland during the second wave included testing of individuals with
Table 1 Study population

\begin{tabular}{lrr}
\hline & \multicolumn{1}{l}{ Cohort } \\
\cline { 2 - 3 } Criterion & $\begin{array}{l}\text { Swiss Fall } \\
\text { 2018 }\end{array}$ & \multicolumn{1}{l}{$\begin{array}{l}\text { Swiss Fall 2020 } \\
\text { COVID-19 }\end{array}$} \\
\hline Alive 1 Oct & 8561495 & $2281790^{*} \dagger$ \\
$\begin{array}{l}\text { SARS-CoV-2 positive } \\
\text { after 1 Oct }\end{array}$ & & $400159^{*}$ \\
35-95 years old & 5179740 & $257445^{*}$ \\
\hline Sex known & & 257400 \\
Not postmortem & & 257288 \\
\hline Study population & 5179740 & 257288 \\
Deaths & 15270 & 5077 \\
\hline
\end{tabular}

Number of individuals in the Swiss Fall 2018 cohort as of 1

October 2018 and total number of 2281790 tests (PCR: 1929095 and antigen: 352 695) in Switzerland between 1 October 2020 and 31 December 2020. The table contains the number of individuals meeting the eligibility criteria in either cohort. 'Study population' refers to the number of observations in either cohort used for the final analysis.

*These numbers were retrieved from official FOPH reports on https://www.covid19.admin.ch/.

†The number of tests specifies the total counts of performed tests including multiple counts for individuals tested more than once. FOPH, Federal Office for Public Health; PCR, Polymerase Chain Reaction.

COVID-19 symptoms (respiratory illness symptoms, high temperature, sudden loss of sense of smell and/or taste, among others). In addition, persons suspected to have been in close contact with a person tested positive for SARS-CoV-2 were also recommended to be tested.

\section{Study population}

The Swiss Fall 2020 COVID-19 cohort included all men and women aged between 35 and 95 years, who tested positive for SARS-CoV-2 during the second wave in Switzerland, starting on 1 October 2020. Individuals who tested positive after their death only were excluded. The Swiss Fall 2018 cohort included all men and women of age 35-95 years alive on 1 October 2018, determined as the number of Swiss inhabitants on 31 December 2018 plus the number of deaths between 1 October 2018 and 31 December 2018 for women and men in the respective age group. This definition assumes that migration during the last 3 months of 2018 did not impact the estimation of mortality.

Eligibility criteria of both cohorts are described in table 1. The Swiss Fall 2014-2017 and 2019 cohorts were defined analogously to the Swiss Fall 2018 cohort in order to assess robustness regarding the selected population in a sensitivity analysis.

\section{Outcome measure}

The outcome measure was time to death due to any cause, measured from a SARS-CoV-2 diagnosis or from 1 October 2018 in the general population. 


\section{Statistical analyses}

In our primary analysis, we assessed the age-specific and sex-specific all-cause mortality of SARS-CoV-2-infected individuals (Swiss Fall 2020 COVID-19 cohort) relative to the general population of 2018 (Swiss Fall 2018 cohort) using survival analysis.

Mortality in both cohorts was analysed adjusted for seasonal effects, by using the same time scale, that is, the number of days after 1 October. For individuals in the Swiss Fall 2020 COVID-19 cohort, a delayed entry approach was used, as infected individuals do not become at risk of death until the date of the SARS-CoV-2 diagnosis. On 31 December, individuals alive in either cohort were administratively right censored.

Sex-specific and age-specific HRs with 95\% CIs were estimated using a stratified Cox proportional hazards model, allowing for separate baseline hazards in the Swiss Fall 2020 COVID-19 and the Swiss Fall 2018 cohorts.

Women aged 65 years were defined as reference and the parameterisation of the linear predictor was chosen to quantify the effects of age and being male in the general population and the additional change in hazard attributable to age and being male in the COVID-19 cohort. $\mathrm{P}$ values and $95 \% \mathrm{CIs}$ for the HRs were adjusted for multiplicity.

To provide a measure of absolute risk, sex-specific and age-specific probabilities of dying within 60 days after start of follow-up were predicted separately for the two cohorts based on estimates from a flexible parametric proportional hazards model. ${ }^{30}$ The model for the Swiss Fall 2020 COVID-19 cohort allows estimation of the sex-specific and age-specific case fatality rate 60 days after a positive test. Relative COVID-19 mortality was defined as the ratio of the probabilities of death with $95 \%$ confidence bands estimated by taking only the uncertainty of the Swiss Fall 2020 COVID-19 cohort into account. ${ }^{31}$

The appropriateness of assuming proportional, linear, and additive effects of age and sex on the hazard function was investigated in three secondary analyses, contrasting the fitted model to models with time-varying sex and age effects, a non-linear additive effect of age on the loghazard scale, or interaction effects of sex and age, respectively (online supplemental material S1). Additionally, we performed a sensitivity analysis by comparing the Swiss Fall 2020 COVID-19 cohort with the Swiss Fall 2014-2017 and 2019 cohorts, defined in an analogous way (online supplemental material S2).

\section{Computational details}

Computations were performed in R V.4.0.4. ${ }^{32}$ To estimate the log-HRs, semiparametric Cox proportional hazards models were fitted using the survival ${ }^{33}$ package. Absolute mortalities were obtained from fully parametric models, parameterised with flexible log-cumulative baseline hazard functions, using the package mlt. ${ }^{30} 34$ Multiplicity-adjusted $\mathrm{p}$ values, CIs and confidence bands were computed using the multcomp package. ${ }^{35}$ Analyses

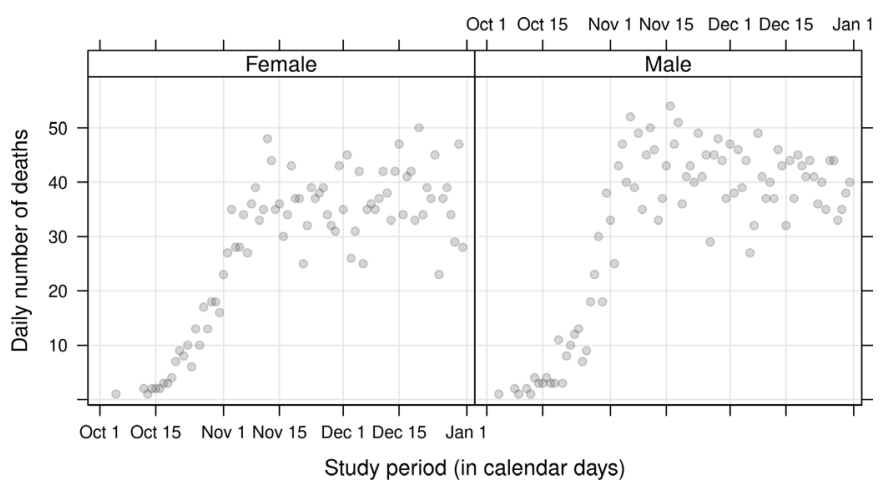

Figure 1 Swiss Fall 2020 COVID-19 cohort. Number of deaths from all causes reported between 1 October 2020 and 31 December 2020 of men and women (aged between 35 and 95 years), which were tested positive for SARS-CoV-2 as of 1 October (excluding postmortem tests).

were independently replicated in $\mathrm{Stata}^{36}$ (V.16), using stpm $2^{3738}$ and merlin ${ }^{39}$.

\section{RESULTS}

Overall, 257288 persons were included in the Swiss Fall 2020 COVID-19 cohort and 5179740 persons in the Swiss Fall 2018 cohort. The second wave in Switzerland was characterised by a steep increase in daily number of deaths, starting from a daily maximum of 5 during the first half of October with peaks of 52-94 deaths in November and December. The daily numbers of deaths among men and women in the Swiss Fall 2020 COVID-19 cohort during the study period from 1 October to 31 December 2020 are shown in figure 1.

Sex and age characteristics of the Swiss Fall 2020 COVID-19 cohort, the Swiss Fall 2018 cohort and the additional Swiss population cohorts of other years (Fall 2014-2017 and 2019) are presented in table 2.

The age distribution of men in the Swiss Fall 2020 COVID-19 cohort matched the age distribution in the Swiss Fall 2018 cohort rather closely (figure 2, right panel), whereas women aged 65-75 years were slightly under-represented in the COVID-19 cohort (figure 2, left panel).

HRs for the effect of male sex and increasing age on all-cause mortality in the Swiss Fall 2018 cohort and additional hazards associated with a SARS-CoV-2 infection in the second wave (Swiss Fall 2020 COVID-19 cohort) are presented in table 3 . In the general population, the all-cause hazard in men was 1.45 (95\% CI: 1.39 to 1.51 ) times the hazard in women. Women aged 65 years experienced the same hazard as men 3.01 years younger. For each additional year of age, the hazard further increased by a factor of 1.13 (95\% CI: 1.13 to 1.13).

The impact of a SARS-CoV-2 infection in the second wave was associated with an increased hazard for men and older patients. Being male with a SARS-CoV-2 infection increased the population hazard by a factor of 1.40 (95\% CI: 1.29 to 1.52 ). Compared with women in the Swiss Fall 2018 cohort, the HR was $1.45 \times 1.40=2.02(95 \%$ 
Table 2 Study populations

\begin{tabular}{|c|c|c|c|c|}
\hline & \multicolumn{2}{|l|}{ Female } & \multicolumn{2}{|l|}{ Male } \\
\hline & $\mathbf{N}$ & Age & $\mathbf{N}$ & Age \\
\hline Fall 2020 COVID-19 & $136110(52.90 \%)$ & $57.21(15.86)$ & $121178(47.10 \%)$ & $56.11(14.42)$ \\
\hline Fall 2019 & 2687464 (51.34\%) & 58.05 (14.95) & $2547327(48.66 \%)$ & $56.43(13.99)$ \\
\hline Fall 2017 & 2635579 (51.40\%) & $57.86(14.92)$ & 2492394 (48.60\%) & $56.16(13.87)$ \\
\hline Fall 2015 & 2578897 (51.46\%) & $57.69(14.89)$ & 2432666 (48.54\%) & $55.88(13.76)$ \\
\hline Fall 2014 & 2549288 (51.52\%) & $57.62(14.89)$ & 2398899 (48.48\%) & $55.77(13.72)$ \\
\hline
\end{tabular}

Number of individuals N (\%) and mean age (SD) for women and men in the Swiss Fall 2020 COVID-19 cohort, the Swiss Fall population cohort of 2018, and earlier cohorts of 2014-2017 and 2019.

CI: 1.91 to 2.14), with more than half of this sex effect of SARS-CoV-2-positive men being attributable to the higher mortality risk of men in the general population (log-HR $0.37+0.34=0.70)$. In comparison with the population age effect, the COVID-19-attributable age effect was very small (HR 1.01, 95\% CI: 1.01 to 1.02), leading to a small increase in the total hazard for each additional year of age (HR: $1.13 \times 1.01=1.14,95 \%$ CI: 1.14 to 1.15 ). Hazards among men in the COVID-19 cohort were comparable with the mortality in women aged 5.24 years older.

The 60-day probability of death from any cause, shown in figure 3 (top) and table 4, was increased for SARS-CoV2-positive individuals compared with the general population. In both cohorts, the 60-day probability of death increased log-linearly in age (figure 3, top), with approximately the same slope, as explained by the very small additional age effect in the Swiss Fall 2020 COVID-19 cohort. Among 100000 individuals, the estimated absolute mortality ranged from 16 (women 35 years old) and 32 (men 35 years old) to 27267 (women 95 years old) and 48002 (men 95 years old) in the Swiss Fall 2020 COVID-19 cohort (table 4). This absolute mortality in the COVID-19 cohort (table 4, second column) can be interpreted as sex-specific and age-specific estimated case fatality rates. Relative COVID-19 mortality ranged from 6-fold to 9-fold for women and from 9-fold to 13-fold for

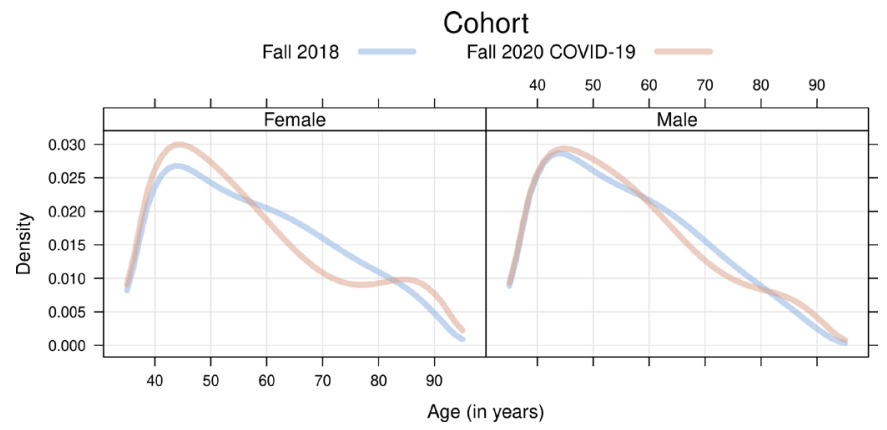

Figure 2 Swiss Fall 2018 and Swiss Fall 2020 COVID-19 cohorts. Comparison of age distribution of women and men in the Swiss Fall 2018 and Swiss Fall 2020 COVID-19 cohorts. men compared with the general population of fall 2018 (figure 3 (bottom panel), table 4), meaning that SARSCoV-2-positive patients were associated with an at least 6 -fold increase and an at most 13-fold increase of the mortality risk when compared with the Swiss Fall 2018 cohort.

Model diagnostics (online supplemental material S1) did not reveal relevant deviations from proportional hazards. The non-linear model indicated a non-linear age effect in the Swiss Fall COVID-19 cohort(online supplemental figures 1 and 2), showing a continuously and monotonically increasing hazard of age. A proportional hazards model allowing sex xage interactions only marginally changed the HRs obtained from the main effects-only model (table 3), however, both the sex and age effects in the Swiss Fall 2020 COVID-19 cohort were attenuated in favour of a small sex xage interaction (online supplemental table 1). Sensitivity analyses (online supplemental material S2) demonstrated that results reported for the Fall 2018 study population were very close to the results for the Fall 2014-2017 and 2019 study populations (online supplemental figures 3-12 and online supplemental table 2).

A direct comparison of the fall mortality patterns for Switzerland with the mortality pattern reported for spring $^{27}$ is possible due to the same study design, study area and statistical analyses techniques employed in both studies. The absolute mortality of men and women in the general population and the COVID-19 cohort of fall 2020 is compared with the corresponding spring cohorts in figure 4 .

In the general population of fall, the absolute mortality was approximately $87 \%$ of the general mortality in spring. Among patients with COVID-19, the reduction of absolute mortality of fall 2020 compared with spring was at least $20 \%$ for older patients and was further reduced for younger patients.

\section{DISCUSSION}

Comparing the all-cause mortality of SARS-CoV-2-positive individuals in the second wave in Switzerland with the 
Table 3 Swiss Fall 2018 and Swiss Fall 2020 COVID-19 cohorts

\begin{tabular}{|c|c|c|c|c|c|}
\hline Effect & log-HR & SE $\times 10$ & P value & HR & $95 \% \mathrm{CI}$ \\
\hline Female & 0 & & & 1 & \\
\hline Male & 0.37 & 0.16 & $<0.001$ & 1.45 & 1.39 to 1.51 \\
\hline Age - 65 & 0.12 & 0.01 & $<0.001$ & 1.13 & 1.13 to 1.13 \\
\hline COVID-19 $\times$ Female & 0 & & & 1 & \\
\hline COVID-19 × Age 65 & 0 & & & 1 & \\
\hline COVID-19 × Age - 65 & 0.01 & 0.02 & $<0.001$ & 1.01 & 1.01 to 1.02 \\
\hline
\end{tabular}

Log-HRs and HRs reflecting the risk of being male ('Male') and each additional year of age ('Age - 65') compared with the baseline hazard among 65-year-old women. The interaction effects describe the additional risk attributable to the SARS-CoV-2 infection. Estimates of log-HRs are presented with SEs and HRs with $95 \%$ Cls. P values and Cls were adjusted for multiplicity.

general population of fall 2018, showed a 6 -fold to 9-fold increase in short-term mortality attributable to a SARSCoV-2 infection for women and 9-fold to 13-fold increase in men. Relatively speaking, all individuals aged between 35 and 95 years suffered roughly the same risk increase. Estimated sex-specific and age-specific case fatality rates ranged between $0.02 \%$ (women, 35 years) and $48.00 \%$
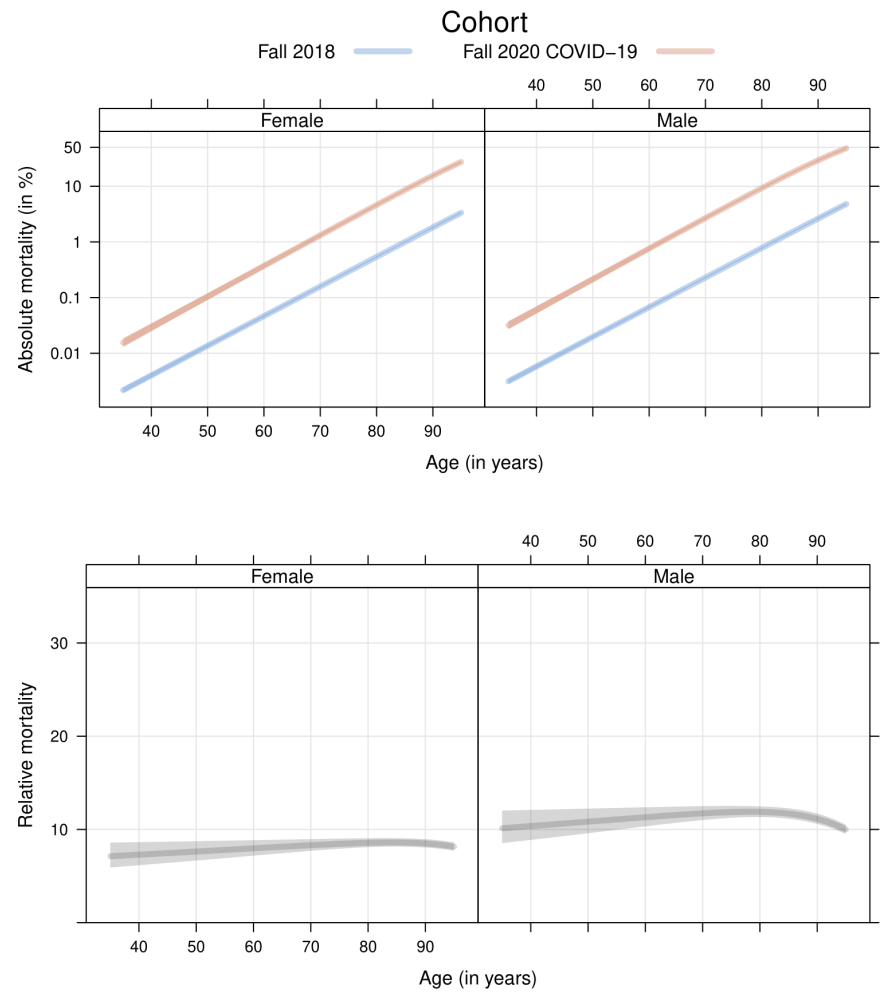

Figure 3 Swiss Fall 2018 and Swiss Fall 2020 COVID-19 cohorts. Absolute mortality, the probability of dying from any cause after 60 days, among women and men of different ages in the Fall 2018 and the Fall 2020 COVID-19 cohorts (top, shown on the logarithmic scale). Relative mortality, the ratio of absolute mortalities in the Fall 2020 COVID-19 and the Fall 2018 cohort, among women and men of different ages (bottom). All estimates are plotted with 95\% confidence bands. (men, 95 years). In the general population not at risk of a SARS-CoV-2 infection, the hazard among men was $45 \%$ larger compared with women, and each additional year of age increased the hazard by $13 \%$. The additional effect of being male in the SARS-CoV-2 positive cohort was $40 \%$; the additional age effect, although statistically significant, was very small.

Our previous study reporting on relative COVID-19 mortality for the first wave in Switzerland in spring $2020^{27}$ was based on the same study design and we are thus able to directly compare the different aspects of burden of COVID-19 between the spring and fall waves. Notably, the total number of deaths in the Swiss Fall 2020 COVID-19 cohort was much higher (with 5077 as compared with 894 in the first wave in spring 2020). The age distribution of infected men in fall 2020 closely matched the 2018 population, whereas larger discrepancies were observed for women in fall, and both sexes in spring.

HRs were almost equivalent to those estimated in the first wave, with a slightly more pronounced male COVID-19 effect and lower associated variability (HR $1.40,95 \%$ CI: 1.29 to 1.52 instead of $1.21,95 \%$ CI: 1.02 to 1.44 in spring).

Absolute mortalities for the spring and fall 2018 populations not at risk of a SARS-CoV-2 infection were slightly smaller in fall, reflecting well-known seasonal patterns of population mortality. ${ }^{40}$ Absolute mortality in the SARSCoV-2 positive cohort, indicated by estimated case fatality rates, decreased at least $20 \%$ compared with spring.

Relative COVID-19 mortalities were lower in fall compared with spring (at least six instead of eight). The much larger sample size in the Swiss Fall 2020 COVID-19 cohort and the larger number of deaths led to a substantial decrease in the variability and the upper confidence limits of the relative mortality.

Testing of more asymptomatic people and systematic mass SARS-CoV-2 testing in some parts of Switzerland may explain the decrease in absolute COVID-19 mortality compared with the first wave in spring 2020, at least in younger patients. 
Table 4 Swiss Fall 2018 and Swiss Fall 2020 COVID-19 cohorts

\begin{tabular}{|c|c|c|c|c|}
\hline \multirow[b]{2}{*}{ Sex } & \multirow[b]{2}{*}{ Age } & \multicolumn{3}{|l|}{ Mortality } \\
\hline & & Absolute Fall 2018 & Absolute SARS-CoV-2 & Relative \\
\hline \multirow[t]{7}{*}{ Female } & 35 & $2(2-2)$ & $16(13-19)$ & $7(6-9)$ \\
\hline & 45 & $7(7-8)$ & $55(47-64)$ & $7(6-9)$ \\
\hline & 50 & $14(13-15)$ & $104(91-119)$ & $8(7-9)$ \\
\hline & 65 & $86(82-90)$ & $700(640-766)$ & $8(7-9)$ \\
\hline & 70 & $159(152-165)$ & $1318(1222-1422)$ & $8(8-9)$ \\
\hline & 75 & $293(282-303)$ & 2474 (2320-2638) & $8(8-9)$ \\
\hline & 80 & $539(522-558)$ & 4620 (4368-4886) & $9(8-9)$ \\
\hline \multirow[t]{9}{*}{ Male } & 35 & $3(3-3)$ & $32(27-38)$ & $10(9-12)$ \\
\hline & 40 & $6(5-6)$ & $60(52-70)$ & $10(9-12)$ \\
\hline & 45 & $11(10-12)$ & $113(99-130)$ & $11(9-12)$ \\
\hline & 50 & $20(18-21)$ & $214(190-242)$ & $11(10-12)$ \\
\hline & 55 & 36 (34-39) & 404 (364-449) & $11(10-12)$ \\
\hline & 60 & $67(64-71)$ & 761 (696-833) & $11(10-12)$ \\
\hline & 65 & $124(119-130)$ & 1433 (1328-1546) & $12(11-12)$ \\
\hline & 70 & $229(221-238)$ & 2688 (2523-2865) & $12(11-12)$ \\
\hline & 75 & $423(408-438)$ & 5015 (4752-5292) & $12(11-13)$ \\
\hline
\end{tabular}

Absolute mortality estimated for the Swiss Fall 2018 (Absolute 2018) and the Swiss Fall 2020 COVID-19 cohort (Absolute SARS-CoV-2, the 60-day sex-specific and age-specific case fatality rate) among 100000 women or men with corresponding age. Relative SARS-CoV-2 mortality (Relative) of the fall was computed as the ratio of the absolute mortalities in the Swiss Fall 2020 COVID-19 compared with the Swiss Fall 2018 cohort. Estimates are shown with Cls obtained from the $95 \%$ confidence bands given in figure 3 .

The share of positive tests among all reported SARSCoV-2 tests ranged between $0 \%$ and $35.9 \%$ during the spring wave and $4.9 \%$ and $30.5 \%$ during the fall wave,

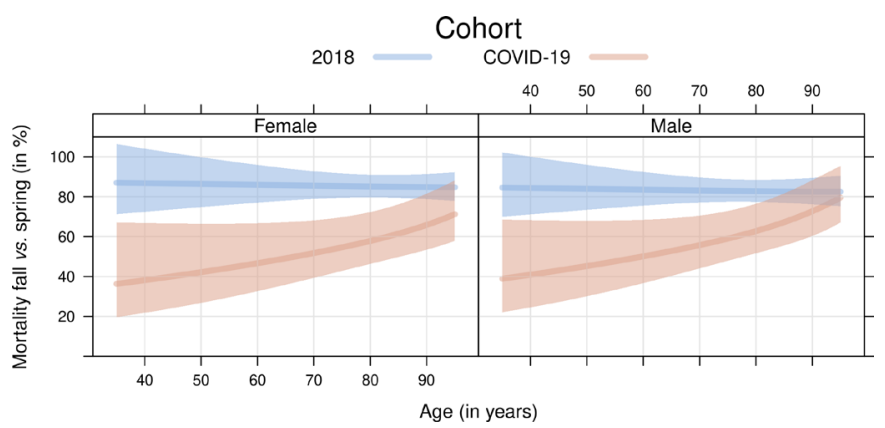

Figure 4 Ratio of absolute mortalities, fall versus spring. Comparison of the mortality of the fall and spring 2020 COVID-19 and general population cohorts. The mortality of men and women in fall compared with the corresponding mortality in spring in either cohort is shown. with the daily trend being increased by $9 \%$ on average during the fall wave (online supplemental figure 13).

Estimates of infection rates from serosurveys indicate that the numbers of undiagnosed COVID-19 cases were larger in the first compared with the second wave with each confirmed SARS-CoV-2 case estimated to represent 2.7 infections in fall compared with 11.6 infections in spring 2020. ${ }^{41}$ Similarly, an analysis from Regensburg, Germany reported higher numbers of asymptomatic cases among the individuals tested positive for SARS-CoV-2 in fall $2020(55.0 \%$ vs $14.4 \%$ in spring $){ }^{42}$ The presence of undiagnosed infections, in spite of broader testing coverage in fall, would suggest an even lower mortality risk than assessed here.

The $20 \%$ reduction of the absolute mortality in fall when compared with spring could be partially explained by the broad use of dexamethasone in Swiss hospitals that has been shown to reduce mortality in severely ill patients with COVID-19. ${ }^{43}$ In addition, also remdesivir 
was used frequently in patients with severe COVID-19 pneumonia in the absence of invasive ventilation. In this group, this intervention also showed a reduction in mortality in one study. ${ }^{44}$ Likely, other improvements in patient management developed based on experiences from the first wave may have also contributed to this finding. ${ }^{45}$

Monitoring of viral variants confirmed that the variant B.1.1.7 (Alpha) constituted a small proportion (weekly average of maximum 2\%) of the SARS-CoV-2 infections during the second wave in Switzerland. No other SARS$\mathrm{CoV}-2$ variants were detected during the study period. ${ }^{46}$

Similar to the comparison of case fatality rates of the first and the second wave among many countries in August $2020,{ }^{21}$ the decreased absolute and relative COVID-19 mortality reported for Switzerland here suggest a potential change in the epidemiological pattern. Broader testing coverage or improved treatment regimes are likely causes but, unfortunately, we can only speculate about their impact on absolute and relative measures of burden of COVID-19. Gaining a better understanding of potential causes and continuous monitoring of mortality might simplify comparisons across settings and allow to monitor the burden of the current pandemic more closely.

\section{Twitter Huldrych Günthard @HGunthard}

Contributors SS, TH, MB and MC designed the study, acquired, preprocessed and analysed the data. SS and TH prepared an initial draft. HG, OK and CEW reviewed results and interpreted the data. All authors contributed to writing the manuscript and approved the final version.

Funding This work was supported by Swiss National Science Foundation under grant number 200021_184603.

Competing interests TH has been paid for consulting, lectures or presentations from Novartis, Roche and PricewaterhouseCoopers. HG has received unrestricted research grants from Gilead Sciences and Roche; fees for data and safety monitoring board membership from Merck; consulting/advisory board membership fees from Gilead Sciences, Merck and ViiV Healthcare; and grants from SystemsX, and the National Institutes of Health. MC has been paid for consulting, lectures or presentations from Roche and Reinsurance Group of America UK Services Limited.

Patient consent for publication Not required.

Ethics approval Patient data were collected by the Swiss Federal Office of Public Health (Bundesamt für Gesundheit) under epidemic law.

Provenance and peer review Not commissioned; externally peer reviewed.

Data availability statement Data and the $\mathrm{R}$ and Stata code to reproduce all results are publically available at https://gitlab.switch.ch/torsten.hothorn/relative_ covid-19_mortality.

Supplemental material This content has been supplied by the author(s). It has not been vetted by BMJ Publishing Group Limited (BMJ) and may not have been peer-reviewed. Any opinions or recommendations discussed are solely those of the author(s) and are not endorsed by BMJ. BMJ disclaims all liability and responsibility arising from any reliance placed on the content. Where the content includes any translated material, BMJ does not warrant the accuracy and reliability of the translations (including but not limited to local regulations, clinical guidelines, terminology, drug names and drug dosages), and is not responsible for any error and/or omissions arising from translation and adaptation or otherwise.

Open access This is an open access article distributed in accordance with the Creative Commons Attribution Non Commercial (CC BY-NC 4.0) license, which permits others to distribute, remix, adapt, build upon this work non-commercially, and license their derivative works on different terms, provided the original work is properly cited, appropriate credit is given, any changes made indicated, and the use is non-commercial. See: http://creativecommons.org/licenses/by-nc/4.0/.
ORCID iDs

Sandra Siegfried http://orcid.org/0000-0002-7312-1001

Torsten Hothorn http://orcid.org/0000-0001-8301-0471

\section{REFERENCES}

1 Odone A, Delmonte D, Scognamiglio T, et al. COVID-19 deaths in Lombardy, Italy: data in context. Lancet Public Health 2020;5:e310.

2 Hauser A, Counotte MJ, Margossian CC, et al. Estimation of SARSCoV-2 mortality during the early stages of an epidemic: a modeling study in Hubei, China, and six regions in Europe. PLoS Med 2020;17:e1003189.

3 Verity R, Okell LC, Dorigatti I, et al. Estimates of the severity of coronavirus disease 2019: a model-based analysis. Lancet Infect Dis 2020;20:669-77.

4 Molenberghs G, Faes C, Aerts J, et al. Belgian covid-19 mortality, excess deaths, number of deaths per million, and infection fatality rates (8 March - 9 May 2020). medRxiv2020.

5 Meyerowitz-Katz G, Merone L. A systematic review and metaanalysis of published research data on COVID-19 infection fatality rates. Int J Infect Dis 2020;101:138-48.

6 Piccininni M, Rohmann JL, Foresti L, et al. Use of all cause mortality to quantify the consequences of covid-19 in Nembro, Lombardy: descriptive study. BMJ 2020;369:m1835.

7 Félix-Cardoso J, Vasconcelos H, Pereira Rodrigues P, et al. Excess mortality during COVID-19 in five European countries and a critique of mortality analysis data. medRxiv2020.

8 Baud D, Qi X, Nielsen-Saines K, et al. Real estimates of mortality following COVID-19 infection. Lancet Infect Dis 2020;20:773.

9 Lipsitch M. Estimating case fatality rates of COVID-19. Lancet Infect Dis 2020;20:775.

10 Ahammed T, Anjum A, Rahman MM, et al. Estimation of novel coronavirus (COVID-19) reproduction number and case fatality rate: a systematic review and meta-analysis. medRxiv2020.

11 Maitra S, Biswas M, Bhattacharjee S. Case-Fatality rate in COVID-19 patients: a meta-analysis of publicly accessible database. medRxiv2020.

12 Lipsitch M, Donnelly CA, Fraser C, et al. Potential biases in estimating absolute and relative case-fatality risks during outbreaks. PLoS Negl Trop Dis 2015;9:e0003846.

13 Rajgor DD, Lee MH, Archuleta S, et al. The many estimates of the COVID-19 case fatality rate. Lancet Infect Dis 2020;20:776-7.

14 Khan JR, Awan N, Islam MM, et al. Healthcare capacity, health expenditure, and civil society as predictors of COVID-19 case fatalities: a global analysis. Front Public Health 2020;8:347.

15 Onder G, Rezza G, Brusaferro S. Case-Fatality rate and characteristics of patients dying in relation to COVID-19 in Italy. JAMA 2020;323:1775-6.

16 Levin AT, Hanage WP, Owusu-Boaitey N, et al. Assessing the age specificity of infection fatality rates for COVID-19: systematic review, meta-analysis, and public policy implications. Eur J Epidemiol 2020;35:1123-38.

17 Jin J-M, Bai P, He W, et al. Gender differences in patients with COVID-19: focus on severity and mortality. Front Public Health 2020;8:152.

18 Spagnolo PA, Manson JE, Joffe H. Sex and gender differences in health: what the COVID-19 pandemic can teach us. Ann Intern Med 2020;173:385-6.

19 Sasson I. Age and COVID-19 mortality: a comparison of Gompertz doubling time across countries and causes of death. Demogr Res 2021:44:379-96.

20 Becher $\mathrm{H}$, Olszewski K, Wiegel S, et al. Changing case fatality risk for COVID-19 over time in selected European countries. medRxiv2020.

21 Fan G, Yang Z, Lin Q, et al. Decreased case fatality rate of COVID-19 in the second wave: a study in 53 countries or regions. Transbound Emerg Dis 2021;68:213-5.

22 Vasconcelos GL, Brum AA, Almeida FAG, et al. Standard and anomalous second waves in the COVID-19 pandemic. medRxiv2021.

23 Leon DA, Shkolnikov VM, Smeeth L, et al. COVID-19: a need for realtime monitoring of Weekly excess deaths. Lancet 2020;395:e81.

24 Pimenoff VN, Elfstrom M, Baussano I, et al. Estimating COVID-19 contribution to total excess mortality. medRxiv2020.

25 Faust JS, Krumholz HM, Du C, et al. All-Cause excess mortality and COVID-19-Related mortality among US adults aged 25-44 years, March-July 2020. JAMA 2021;325:785-7.

26 Woolf SH, Chapman DA, Sabo RT, et al. Excess deaths from COVID-19 and other causes, March-April 2020. JAMA 2020;324:510-3. 
27 Hothorn T, Bopp M, Günthard H, et al. Assessing relative COVID-19 mortality: a Swiss population-based study. BMJ Open 2021;11:e042387.

28 Kontis V, Bennett JE, Rashid T, et al. Magnitude, demographics and dynamics of the effect of the first wave of the COVID-19 pandemic on all-cause mortality in 21 industrialized countries. Nat Med 2020;26:1919-28.

29 Bundesamt für Gesundheit (Swiss Federal Office of Public Health, FOPH). Coronavirus: tests. technical report, 2020. Available: https://www.bag.admin.ch/bag/en/home/krankheiten/ausbruecheepidemien-pandemien/aktuelle-ausbrueche-epidemien/novel-cov/ testen.html [Accessed 11 May 2020].

30 Hothorn T, Möst L, Bühlmann P. Most likely transformations. Scandinavian Journal of Statistics 2018;45:110-34.

31 Ederer F, Axtell LM, Cutler SJ. The relative survival rate: a statistical methodology. Natl Cancer Inst Monogr 1961;6:101-21.

32 R Core Team. R: a language and environment for statistical computing. Vienna, Austria: R Foundation for Statistical Computing, 2020. http://www.R-project.org/

33 Therneau TM. survival: survival analysis. R package version 3.2-7, 2020. Available: https://CRAN.R-project.org/ package=survival

34 Hothorn T. Most Likely Transformations: The mlt Package. J Stat Softw 2020;92:1-68.

35 Hothorn T, Bretz F, Westfall P. multcomp: simultaneous inference in general parametric models. R package version 1.4-16, 2021. Available: https://CRAN.R-project.org/package=multcomp

36 StataCorp. Stata statistical software: release 16. College Station, TX, U.S.A: StataCorp, 2019.

37 Royston P, Parmar MKB. Flexible parametric proportional-hazards and proportional-odds models for censored survival data, with application to prognostic modelling and estimation of treatment effects. Stat Med 2002;21:2175-97.

38 Lambert PC, Royston P. Further development of flexible parametric models for survival analysis. Stata J 2009;9:265-90.

39 Crowther MJ. merlin-A unified modeling framework for data analysis and methods development in Stata. Stata $J$ 2020;20:763-84.

40 Bundesamt für Statistik (Swiss Federal Statistical Office, FSO). Deaths per month and mortality since 1803. technical report, 2021. Available: https://www.bfs.admin.ch/bfs/en/home/ statistiken/ kataloge-datenbanken/daten.assetdetail.14387168.html [Accessed 26 Feb 2021]

41 Stringhini S, Zaballa M-E, Perez-Saez J, et al. Seroprevalence of antiSARS-CoV-2 antibodies after the second pandemic peak. Lancet Infect Dis 2021;21:600-1.

42 Lampl BM, Salzberger B. Changing epidemiology of COVID-19. GMS Hygiene and Infection Control 2020;15

43 The RECOVERY Collaborative Group. Dexamethasone in hospitalized patients with Covid-19. N Engl J Med Overseas Ed 2021;384:693-704.

44 Beigel JH, Tomashek KM, Dodd LE, et al. Remdesivir for the treatment of Covid-19 - final report. N Engl J Med Overseas Ed 2020;383:1813-26.

45 Karagiannidis C, Windisch W, McAuley DF, et al. Major differences in ICU admissions during the first and second COVID-19 wave in Germany. Lancet Respir Med 2021;9:e47-8.

46 Bundesamt für Gesundheit (Swiss Federal Office of Public Health, FOPH). COVID-19 Switzerland: relevant virus variants. technical report, 2021. Available: https://www.covid19.admin.ch/en/ epidemiologic/virus-variants [Accessed 11 Jun 2021] 